\title{
Creatividad y transferencia didáctica en la acción pedagógica de docentes universitarios chilenos $i$
}

\author{
Creativity and didactic transfer in the pedagogical action \\ of Chilean university professors
}

\section{Criatividade e transferência didática na ação pedagógica de professores universitários chilenos}

\author{
ISSN 1688-9304 - DOI: 10.18861/cied.2020.11.2.2992
}

Alejandro Villalobos Claveria*1

https://orcid.org/0000-0003-1440-9942

Yenia Melo Hermosilla**2

https://orcid.org/0000-0003-4120-9452

Fecha de recibido: 21/05/2019

Fecha de aprobado: 29/03/2020

\section{Resumen}

Este artículo analiza experiencias de transposición didáctica como expresión creativa de la práctica pedagógica de académicos universitarios chilenos. Se asumió un enfoque cuantitativo de naturaleza descriptiva y analítica a una muestra intencional de 59 docentes de tres universidades regionales chilenas a quienes se aplicó una pauta de observación de clases durante el periodo 2014-2015. Los resultados indican que existen dos aspectos de la creatividad que se expresan en la transposición didáctica: la utilización de recursos didácticos novedosos y la relación que establecen entre su enseñanza y los problemas de la disciplina o futura profesión de los estudiantes. Se concluye que el profesor, en su didáctica, en busca de innovación utiliza procesos creativos. Sin embargo, en la evaluación del aprendizaje tiende a ser reproductivo debido a la exigencia del marco curricular vigente.

Palabras clave: prácticas de enseñanza, creatividad, transferencia didáctica, profesores universitarios.

\begin{abstract}
This article analyzes experiences of didactic transposition, as a creative expression of the pedagogical practice carried out by Chilean university academics. A quantitative approach of a descriptive and analytical nature was conducted to a purposive sample of 59 teachers from three Chilean regional universities, to whom a pattern of class observation was applied during the period 2014-2015. The results indicate that there are two aspects of creativity that are expressed in the didactic transposition: the use of innovative didactic resources and the relationship they establish between their teaching and the field matters or future profession of students. It is concluded that in their didactics teachers use creative processes, as they seek innovation, while in the assessment of learning, the trend is reproductive, due to the requirement of the current curricular framework.
\end{abstract}

Keywords: educational practices, creativity, didactic transference, university teachers. 


\section{Resumo}

Este artigo analisa experiências de transposição didática, como expressão criativa da prática pedagógica de acadêmicos universitários chilenos. Foi adotada uma abordagem quantitativa, descritiva e analítica para uma amostra intencional de 59 professores de três universidades regionais chilenas, aplicando-se, para tanto, um padrão de observação de aula, durante o período 2014-2015. Os resultados indicam que existem dois aspectos da criatividade que se expressam na transposição didática: o uso de recursos didáticos inovadores e a relação que eles estabelecem entre seu ensino e os problemas da disciplina (conteúdo) ou a futura profissão dos alunos. Conclui-se que o professor utiliza processos criativos em seu ensino, buscando a inovação didática, mas na avaliação da aprendizagem ele é propenso a ser reprodutivo, devido às exigências do atual quadro curricular.

Palavras-chave: práticas de ensino, criatividade, transferência didática, professores universitários.

\section{Introducción}

Hoy día existe consenso en el concierto nacional e internacional sobre la necesidad de desarrollar el pensamiento creativo para cambiar, innovar o transformar el mundo social, cultural y productivo de las naciones (Unesco, 2005; Drucker, 1992). “En las sociedades del conocimiento, los valores y prácticas de creatividad e innovación desempeñarán un papel importante [...] para responder mejor a las nuevas necesidades de la sociedad. La creatividad y la innovación conducen asimismo a promover procesos de colaboración de nuevo tipo" (Unesco, 2005, p.19). En este sentido se tiende a aceptar que "El saber está deviniendo rápidamente el factor número uno de la producción, desplazando capital y mano de obra a un segundo plano" (Drucker, 1992). En esta misma línea ninguna persona, institución o empresa puede sustraerse al imperativo categórico que se promueve en este siglo XXI de que el conocimiento y los saberes especializados desempeñan un papel fundamental en dicho cambio. Así, por ejemplo, la formación profesional superior se encuentra demandada por la sociedad en su conjunto para responder a la formación de profesionales capaces y creativos que son requeridos por el mercado del trabajo (Drucker, 1992).

Los docentes de la educación superior se ven enfrentados al desafío no solo de incorporar en sus prácticas de enseñanza el dominio del contenido de la disciplina en sí sino que además deben desarrollar competencias asociadas a la apropiación de conocimiento didáctico para vehiculizar el saber disciplinario hacia el saber didáctico que se requiere con el fin de cumplir con el acto educativo planificado (Pellón, Mansilla y San Martín, 2009; Pérez Lillitsy, Massón Cruz y Torres Miranda, 2020). Por cierto, este es el núcleo del trabajo de una transferencia didáctica.

De algún modo se devuelve el rol protagónico al profesor cuando se exige una gestión adecuada del proceso de enseñanza-aprendizaje que asegure aprendizajes eficientes y una educación de calidad (Bolívar Botía y Bolívar Ruano, 2012) pues esta labor docente es la base de este mejoramiento y representa una tarea clásica de la didáctica en la que el aula es el espacio privilegiado para dicha optimización. Por cierto, se reconoce que el profesorado constituye un componente esencial y su actuación puede ser un factor crítico o clave para la transformación de la vida en las aulas. 
Entre el proyecto curricular diseñado y las prácticas docentes sugeridas se encuentra el profesor como el agente que modela y reconstruye los saberes que confluyen en su clase mediante su propia apropiación didáctica la que, posteriormente, ofrece a sus estudiantes en su exposición oral, trabajo guiado, evaluaciones, textos de apoyo, entre otros (Bolívar Botía y Bolívar Ruano, 2012).

Interesa destacar que las interacciones docente-alumno constituyen el elemento central del proceso enseñanza-aprendizaje, fenómeno complejo y global que ocurre como expresión material de la vida en las aulas. Para algunos autores (Jackson, 2009, entre otros) la vida de las aulas es un campo de problematización permanente pues en ella entran en conflicto los intereses y necesidades de sus actores que, a menudo, se encuentran en posiciones opuestas (profesores y alumnos). Por ende, llegar a comprender la vida y el trabajo del aula supone reconocer que todos los días y en cada una de las clases entran en interacción diferentes culturas (profesores y alumnos como unidades sociales) pero también acciones humanas provenientes de códigos pedagógicos, organizativos y sociales que impone la propia institución educativa (Chevallard, 1991).

Lo anterior representa el escenario de la presente investigación, donde docentes y estudiantes universitarios confluyen en el aula como participantes de un proceso de formación superior pues allí convergen múltiples y complejas interacciones. Cada aula es un escenario en el que interactúan diferentes variables didácticas que hacen de la enseñanza y el aprendizaje actos complejos y desafiantes en su comprensión. En este núcleo didáctico se produce y ancla la posibilidad del mejoramiento de una educación de calidad, y en las interacciones efectivas en una tríada didáctica conformada por profesores, alumnos y contenidos en la sala de clases se evidencia la oportunidad de lograr un aprendizaje de calidad (Elmore, 2010).

\section{La transposición didáctica}

En las últimas décadas, y particularmente en el campo de la didáctica general, ha tomado fuerza el concepto de transposición didáctica como el enfoque explicativo del tránsito de un saber sabio al saber enseñado, es decir, a la transformación del saber experto en un saber didáctico pasible de ser aprendido por un alumno y dentro de un contexto dado (Brousseau, 2011; Chevallard, 1991; De Camilloni, Cols, Basabe \& Feeney, 2007).

Tal como ocurre en el contexto escolar, en el ámbito universitario circulan diversos saberes que se van constituyendo según las dinámicas de la enseñanza. La enseñanza universitaria, entonces, se encarga de posibilitar que esos saberes sabios se constituyan en saberes por enseñar, en saberes enseñados, en saberes por aprender y en saberes aprendidos, para producir la transposición didáctica (González y Díaz, 2008). En este proceso didáctico hay un trabajo de transformación que es el cambio que experimenta el objeto del saber al ser reconstruido en el aula de manera intersubjetiva entre el profesor que domina los conocimientos de su disciplina y sus alumnos, quienes ya cuentan con un saber experiencial (Pellón, Mansilla y San Martín, 2009). La clase constituye el lugar donde se tematiza el saber sabio y es el objeto de estudio de la metódica, campo de la didáctica. Sin embargo, para que el saber por enseñar llegue hasta la clase, tanto ese saber como la clase necesitan ser pensados desde la didáctica, es decir, desde una teoría acerca de las prácticas de la enseñanza (Litwin, 2001; Martínez y González, 2009).

A través de la transposición didáctica el docente tratará de mediar en los aprendizajes de los estudiantes ubicando el conocimiento dentro del contexto socio-histórico-cultural 
que rodea a la nueva situación de aprendizaje, así como intentará elaborar un discurso comprensible para el estudiante. Para lograr este propósito el docente descontextualizará, despersonalizará y destemporalizará el saber sabio para generar este nuevo saber didáctico: el objeto de enseñanza (Chevallard, 1991).

Este proceso de transposición didáctica, entonces, tiene pretensión de validez universal en la medida de que asume que el saber pedagógico mantiene una dependencia epistemológica con el saber experto, donde el profesor cautela su validez y aplicabilidad curricular para un mejor aprendizaje de los alumnos.

No obstante debe tenerse en cuenta la existencia de las teorías implícitas, prácticas pedagógicas y aspectos éticos que guían las acciones del docente universitario (BastoTorrado, 2011). Al mismo tiempo, se deben considerar las concepciones previas del alumno, lo que posibilita que la reconstrucción del objeto de enseñanza se torne significativa para docentes y estudiantes. La interacción didáctica de ambos participantes, desde sus propias experiencias de elaboración de conocimiento, se articula en un proceso común de resignificación. Dichas aportaciones confluyen en un proceso que adquiere pretensiones de validez intersubjetivas tanto para el profesor como para sus alumnos; situación que no está exenta de creatividad por parte del propio docente. (Basto-Torrado, 2011).

En suma, reconocer el lugar privilegiado que ocupa el aula como un espacio de acción interactiva y construcción práctica entre docentes y estudiantes ayuda a comprender parte del complejo proceso de transformaciones adaptativas del saber experto que se expresa en la transposición didáctica.

A través de los mecanismos de la transposición didáctica que ocurren en el aula se generan y desarrollan los procesos de enseñar y de aprender, verdadero espacio de realización personal y social del profesor y sus estudiantes y que requiere de alguna dosis de creatividad por parte del propio docente.

\section{Creatividad y educación}

En la actualidad la creatividad tiene una gran importancia como eje transversal de diferentes actividades humanas, principalmente dentro de la educación y especialmente para la enseñanza y se reconoce, asimismo, su nueva relevancia para la educación superior (Alonso y Arandia, 2014; Boden, 1994; Duarte, 1998; Elisondo, 2015; Sternberg y Lubart, 1997; Marina, 2011).

Existen, al menos, tres motivos para justificar una mirada creativa de la educación: por el impacto positivo que la creatividad tiene en la vida de las personas, por las posibilidades que generan las innovaciones educativas tanto en los alumnos como en el profesorado y, fundamentalmente, por ser la creatividad una capacidad aplicable a diferentes contextos, niveles y situaciones sociales, culturales y productivas (De la Torre, 2006).

La complejidad del fenómeno educativo obliga a aceptar una mirada global y distinta de sus participantes. No hay que olvidar que los problemas complejos solo pueden resolverse mirándolos desde diferentes puntos de vista y desde marcos analíticos heterogéneos. Estimular formas divergentes de pensamiento, de resolución de problemas y de toma de decisiones es una acción educativa socialmente significativa (Alonso y Arandia, 2014; Corbalán, Martínez y Donolo, 2003; De la Torre, 2013; Elisondo, 2015). 
En la educación universitaria la creatividad se convierte en una competencia esencial en muchos perfiles de egreso profesional, con el fin de lograr un posterior desarrollo eficaz en el desempeño laboral. Desde este punto de vista los docentes universitarios están llamados a crear entornos educativos que favorezcan la adquisición y desarrollo de la capacidad creativa como habilidad transversal en la formación profesional (Alonso y Arandia, 2014; Martínez y González, 2009).

Pensar la educación desde una perspectiva creativa implica divergencias, flexibilidad y alternativas a la hora de pensar las propuestas pedagógicas, los contenidos y los encuadres didácticos. La creatividad se vincula con la posibilidad de generar ideas y productos originales e innovadores que generen rupturas en los estándares y en las formas rutinarias de actuación (Corbalán, Martínez y Donolo, 2003; Csikszentmihalyi, 1998; De la Torre y Violant, 2006; Elisondo, 2015; Alonso, 2001; Sternberg y Lubart, 1997).

En este contexto se destaca la necesidad de potenciar la creatividad como competencia universitaria de tipo sistémico, es decir, que para el desarrollo de las capacidades creativas en el estudiante es necesario la implicación de otras competencias y viceversa, es decir, otras competencias precisan de la capacidad creativa para ser desarrolladas. Para ello es necesario crear un contexto educativo favorable a los cambios, la innovación, la investigación y la experimentación (Finke, Ward y Smith, 1996; Martínez y González, 2009; Palomares, 2011).

En esta línea de pensamiento De la Torre y Violant (2013) postulan que, a través de las estrategias creativas del docente el estudiante adquiere un protagonismo mayor que en las metodologías tradicionales, es decir, a través de la aplicación de estrategias didácticas creativas el alumno construye los conocimientos y desarrolla habilidades mediante la búsqueda personal orientada por el profesor. Esta situación permite que el aprendizaje sea más atrayente y motivador para todos los participantes del proceso educativo.

\section{Creatividad y transposición didáctica. Un puente por construir}

Establecer una vinculación entre los conceptos centrales del presente artículo resulta una tarea ardua y laboriosa que escapa a la naturaleza de este trabajo. No obstante plantearemos algunos conceptos e ideas que tienden a legitimar dicha conexión a fin de comprender la actuación docente del profesor universitario no pedagogo. Cabe señalar que la expresión docente no pedagogo debe entenderse como un profesional que ejerce la docencia sin tener formación específica o capacitación previa en este ámbito. Por cierto, aceptar esta definición operacional del citado concepto permite una mayor comprensión de la naturaleza del quehacer del docente de la educación superior y sus desafíos pedagógicos cotidianos (Villalobos Claveria y Melo Hermosilla, 2019).

En este sentido, y dentro de la elaboración conceptual de la relación entre creatividad y transposición didáctica puede ser interesante tener presente estas dos ideas previas:

a) Existen teorías y creencias implícitas en los profesores que se orientan y se reconocen en su quehacer docente.

b) Los alumnos disponen de ideas previas que expresan como significados conceptuales para organizar su aprender. 
Frente a estas premisas conviene plantearse las siguientes interrogantes: ¿Cómo se puede despertar la creatividad de los alumnos? ¿Qué debe pensar el docente que permita estimular el pensamiento creativo de sus alumnos? ¿Qué debe hacer la educación para lograr alumnos creativos e innovadores?

Por ende, para que la didáctica favorezca un aprendizaje creativo debe tender un puente emancipador entre la visión epistemológica del conocimiento específico y los procesos de aprendizaje que llevan a cabo los alumnos. La travesía por este puente implica que en la acción educativa entre profesor y alumnos la generación del conocimiento tenga una doble dimensión: por su lado los educandos reconstruyen el conocimiento científico, literario, lingüístico y matemático entre otros, y por el suyo el docente reconstruye el conocimiento didáctico respecto de cómo vincular las explicaciones epistemológicas del conocimiento con los procesos que posibilitan su aprendizaje. Esto le permite reforzar sus teorías implícitas o bien le facilita su movilización al punto de problematizarlas transformativamente para, desde allí, plantearse un proceso creativo de reelaboración del saber a través del desempeño de una práctica reflexiva (De la Torre, 2006; Perrenoud, 2010; Tardif, 2004).

Es una situación desafiante para la didáctica en tanto puente epistemológico pues no solo debe poseer una naturaleza emancipadora para los alumnos sino también para el profesor, quien debe ser capaz de provocar la búsqueda de una expresión creativa de su saber (De la Torre y Violant, 2013; Palomares, 2011).

En suma, la transposición didáctica tiene más de una vía de concreción para favorecer la enseñanza y el aprendizaje creativos tanto para el profesor como para el alumno. No obstante, la elaboración de este conocimiento debe darse en el desarrollo de un proceso de aprendizaje que convoque a la creatividad como un elemento central pues la educación la reclama como una entidad protagónica para el desarrollo de nuestra sociedad, desafío que puede ser abordado desde la práctica docente (Medina Moya y Jarauta Borrasca, 2012)

De este modo se reconoce que la creatividad es una de las capacidades humanas más importantes que debe desarrollar la educación, tanto en los docentes como en los alumnos ya que con ella el hombre transforma y se transforma, con ella el hombre hace cultura. Este resultado permite señalar que la creatividad es uno de los rasgos más relevantes a desarrollar en la educación contemporánea, particularmente en la formación profesional universitaria actual.

\section{Materiales y métodos}

La presente investigación se adscribe a un enfoque cuantitativo y busca describir y cuantificar la naturaleza de las interacciones didácticas que se producen en el aula universitaria mediante la identificación de dos variables que interactúan entre sí según lo señalado en el marco conceptual del presente trabajo. Se trata de una investigación descriptiva que mediante la observación estructurada pretende analizar la relación entre creatividad y transposición didáctica que, eventualmente, surge en el aula universitaria. El objetivo que constituye el eje de esta etapa de la investigación es el siguiente:

- Cuantificar y analizar experiencias de transposición didáctica que se evidencian en las actuaciones de docentes no pedagogos en la formación profesional universitaria en tres universidades regionales, desde la perspectiva de la creatividad. 
En tanto técnica investigativa, la observación de clases permitió recoger evidencia en torno a la práctica docente en el aula con el propósito de reconocer los procesos asociados a la transposición didáctica. La elección de esta perspectiva epistemológico- metodológica se justifica por cuanto se busca lograr una descripción, codificación, cuantificación y análisis del comportamiento docente en la enseñanza universitaria (Hernández Sampieri et al., 2014).

El objeto de estudio está constituido por los docentes no pedagogos de las principales universidades chilenas del Consejo de Rectores de la región del Biobío que imparten asignaturas en el área de formación profesional superior. Se exceptúan los profesionales que cuentan con formación sistemática en pedagogía y los docentes de las carreras del área de educación.

La docencia que realizan aquellos docentes corresponde a carreras y/o programas de postgrado vinculados a las áreas de ciencias experimentales, ciencias sociales y ciencias de la salud. Esta clasificación laboral de los docentes surge según sea la aplicación del método científico al tipo de fenómeno que estudia y al tipo de propósito que guía el quehacer investigativo de cada ciencia. Cabe señalar que dicha clasificación se apoya en la que realiza Mario Bunge (1996) de las ciencias así como en lo expresado por Lamo de Espinosa, González García y Torres Albero (2007, cap. 3) quienes señalan la diferencia entre conocimiento teórico y conocimiento práctico, saber fáctico y saber valorativo y donde, asimismo, se expone el valor y la utilidad del conocimiento en el desarrollo cultural de la humanidad.

La conformación de la muestra fue de naturaleza intencional (selección no probabilística) pero buscando la representatividad de la población en estudio. En la elección de los sujetos participantes no hay criterios asociados a género, dependencia administrativa o jerarquía académica. Una posterior agrupación sí fue realizada en base a pertenencia a universidad de origen y a jerarquía académica a fin de facilitar un análisis interno por universidad y años de experiencia.

Se establecen, entonces, tres grupos provenientes de cada universidad participante (Universidad de Concepción, Católica de Concepción y del Biobío) organizados en tres categorías según años de experiencia. Se fundamenta esta elección en la variable Años de experiencia docente (entendiéndose por docente novel a aquel con tres años de experiencia como profesor universitario pero menos de cinco; por docente en desarrollo a aquel con más de cinco años pero menos de 20 de experiencia docente y por docente experto a aquel con 20 años o más de experiencia académica).

La naturaleza de la variable tiempo responde a la evaluación que se realiza en la universidad chilena según la cual un docente novel tiene su primera evaluación entre los tres y los cinco años de trabajo. Por su parte, un docente en desarrollo ya ha sido evaluado más de una vez y ha recibido retroinformación sobre su desempeño. Finalmente, el docente experto se encuentra consolidado en su universidad como resultado de evaluaciones y de suficiente retroalimentación que le ha permitido un mejoramiento y ascenso académicos. Para la recolección de datos fue elaborada una pauta estructurada de la observación en aula. Dicha pauta fue aplicada a los sujetos seleccionados de las áreas de ciencias experimentales, ciencias sociales y ciencias de la salud. Esta distribución fenoménica se resume en la siguiente tabla. 
Tabla 1. Cuadro resumen del tamaño de la muestra por años de experiencia docente y cantidad de observaciones realizadas en cada universidad.

\begin{tabular}{|l|c|c|c|c|c|c|c|c|c|c|}
\hline \multirow{2}{*}{$\begin{array}{l}\text { Instrumento } \\
\text { a aplicar }\end{array}$} & \multicolumn{4}{|c|}{ Universidad 1 } & \multicolumn{3}{c|}{ Universidad 2 } & \multicolumn{3}{c|}{ Universidad 3 } \\
\cline { 2 - 10 } & Novel & $\begin{array}{c}\text { En } \\
\text { desarrollo }\end{array}$ & Experto & Novel & $\begin{array}{c}\text { En } \\
\text { desarrollo }\end{array}$ & Experto & Novel & $\begin{array}{c}\text { En } \\
\text { desarrollo }\end{array}$ & Experto & Total \\
\hline $\begin{array}{l}\text { Observación } \\
\text { de clase }\end{array}$ & 18 & 24 & 15 & 12 & 29 & 17 & 18 & 24 & 18 & 175 \\
\hline Docentes & 6 & 8 & 5 & 4 & 10 & 6 & 6 & 8 & 6 & 59 \\
\hline
\end{tabular}

Cabe señalar que la construcción de esta pauta obedece a categorías teóricas de la creatividad sustentadas por Csikszentmihalyi (1998) y De la Torre y Violant (2013) así como por las ideas propuestas por Chevallard (1991) sobre transposición didáctica. En la redacción de los ítems se procuró que ambos enfoques pudieran ser reconocidos en la observación de aula.

Posteriormente esta pauta de observación de clase fue validada por cinco jueces expertos en evaluación educacional y psicometría (dos psicólogos y tres profesores evaluadores de universidades externas a la muestra consultada) quienes juzgaron la pertinencia de los ítems así como la aplicabilidad y la claridad de su redacción. Se conformó una lista definitiva de siete indicadores. Estos reactivos asociados a los indicadores de creatividad que pueden reconocerse en las prácticas docentes se presentan en la siguiente tabla.

Tabla 2. Pauta de observación, categoría creatividad y sus respectivos indicadores.

\begin{tabular}{|c|c|}
\hline Categoría & Indicadores \\
\hline \multirow{7}{*}{ 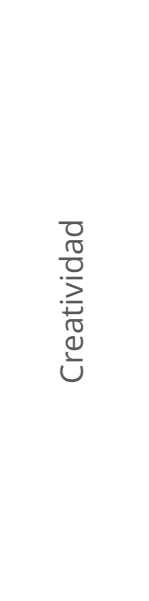 } & $\begin{array}{l}\text { CR 1: El profesor comparte los resultados de una actividad exitosa (investigación, } \\
\text { ponencia, entre otros) con sus alumnos para que vean si la pueden replicar en sus } \\
\text { estudios. }\end{array}$ \\
\hline & $\begin{array}{l}\text { CR 2: El profesor utiliza diversos medios para generar una clase original (creativa) e } \\
\text { innovadora para sus alumnos. }\end{array}$ \\
\hline & CR 3: El docente plantea preguntas divergentes o fecundas en sus resultados. \\
\hline & $\begin{array}{l}\text { CR 4: El docente promueve el cambio en la perspectiva de comprender los temas } \\
\text { planteados. }\end{array}$ \\
\hline & $\begin{array}{l}\text { CR 5: El profesor ofrece distintas formas (o alternativas) de elaborar los trabajos por } \\
\text { hacer. }\end{array}$ \\
\hline & CR 6: El profesor utiliza recursos estimuladores de la imaginación y la fantasía. \\
\hline & $\begin{array}{l}\text { CR 7: El profesor vincula de forma novedosa su enseñanza con problemas de la } \\
\text { disciplina y/o con la futura profesión del estudiante. }\end{array}$ \\
\hline
\end{tabular}

La presencia o ausencia de estos indicadores en las prácticas docentes se registró con los indicadores se observa o no se reconoce respectivamente. La aseveración no corresponde fue consignada cuando situaciones particulares de las prácticas docentes no permitieron la manifestación de dicho indicador.

A través del trabajo de campo se obtuvo una muestra total de 59 docentes no pedagogos pertenecientes a tres universidades de la Región del Biobío quienes se distribuyeron por categoría y dependencia institucional y que generaron un total de 175 observaciones realizadas en este estudio de campo (véase anterior tabla 1). 
Las pautas de observación aplicadas a cada docente de la muestra fueron procesadas con Microsoft Excel y sistematizadas bajo la lógica de la presencia versus ausencia de cada indicador determinado. Este procedimiento se aplicó a la totalidad de la muestra.

Finalmente cabe advertir que en las aulas universitarias -ya sea por el elevado número de estudiantes o por su infraestructura inadecuada- el trabajo colaborativo se ve limitado o es imposible desarrollarlo. Esto puede impedir la manifestación y, por lo tanto, la observación de determinados indicadores de creatividad. En esta misma línea, De la Torre y Violant (2013) se refieren al carácter colaborativo o compartido del conocimiento en el que se puede confrontar la información favoreciendo la enseñanza creativa. Por dicha razón no fueron considerados indicadores asociados al trabajo grupal facilitador de una expresión creativa.

\section{Resultados y discusión}

Los resultados de las observaciones de las prácticas pedagógicas fueron agrupados e interpretados por cada universidad participante con el propósito de obtener una mirada individual de institución en estudio. Seguidamente se realizó un análisis global de la muestra en estudio con la intención de hallar tendencias en sus resultados.

Cabe señalar que no se realizó un análisis específico por cada categoría docente o por años de experiencias de los docentes consultados (nóveles, en desarrollo y expertos) pues dichos resultados no eran diferentes en estas categorías de sujetos consultados. Al realizar una selección y clasificación de estos sujetos, no obstante, se había esperado obtener resultados diferentes en cada estrato.

Sin embargo en un análisis preliminar se hallaron diferencias entre las universidades consultadas. Esta situación explica estas decisiones metodológicas realizadas, tanto para su análisis e interpretación como en las conclusiones obtenidas.

\section{Análisis individual de cada caso Universidad 1}

La categoría creatividad y sus respectivos indicadores asociados a la transposición didáctica que se reconocen en esta acción docente pueden observarse en la tabla 3 y en la figura 1.

Las actividades creativas más frecuentes con un $84 \%$ y $79 \%$ respectivamente corresponden al planteamiento de preguntas divergentes en sus resultados y a la promoción del cambio en la perspectiva de comprensión de los temas planteados en clases. Por su parte, con una menor frecuencia (74\%) dichas actividades vinculan de forma novedosa su enseñanza con problemas de la disciplina o de la futura profesión del estudiante. Finalmente, la oferta de distintas opciones a los estudiantes para que elaboren los trabajos de la asignatura se destaca por su bajo porcentaje (un 32\% de las observaciones). 
Tabla 3. Frecuencia de indicadores de creatividad en la Universidad 1.

\begin{tabular}{|l|c|c|c|c|c|c|c|}
\hline \multicolumn{2}{|c|}{ Indicadores de creatividad } \\
\hline & CR1 & CR2 & CR3 & CR4 & CR5 & CR6 & CR7 \\
\cline { 2 - 8 } & Porcentaje & Porcentaje & Porcentaje & Porcentaje & Porcentaje & Porcentaje & Porcentaje \\
\hline Se observa & 42.0 & 58.0 & 84.0 & 79.0 & 32.0 & 63.0 & 74.0 \\
\hline $\begin{array}{l}\text { No se } \\
\text { reconoce }\end{array}$ & 53.0 & 37.0 & 11.0 & 21.0 & 47.0 & 37.0 & 26.0 \\
\hline $\begin{array}{l}\text { No } \\
\text { corresponde }\end{array}$ & 5.0 & 5.0 & 5.0 & 0.0 & 21.0 & 0.0 & 0.0 \\
\hline Total & 100.0 & 100.0 & 100.0 & 100.0 & 100.0 & 100.0 & 100.0 \\
\hline
\end{tabular}

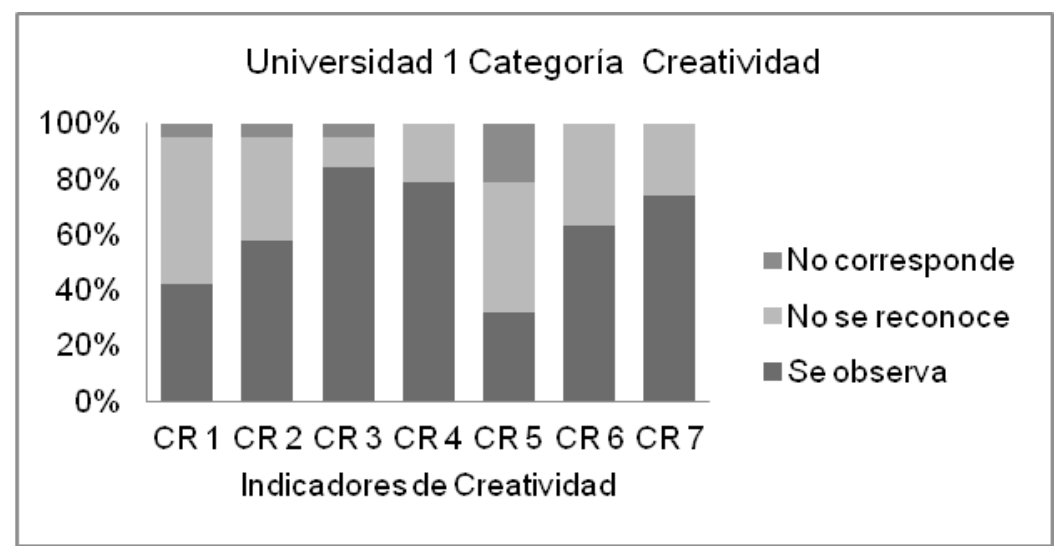

Figura 1. Indicadores de creatividad y frecuencia observados en la acción docente

\section{Universidad 2}

La mayoría de los docentes observados desarrolla con más frecuencia cuatro acciones vinculadas a la creatividad que se encuentran asociadas a la transposición didáctica, lo cual se puede observar en la tabla 4 y en la figura 2. Las acciones que destacan en este grupo de docentes son el planteamiento de preguntas divergentes en sus resultados (84\%), seguido de los siguientes indicadores que se concretaron en el $74 \%$ de las observaciones de aula:

- Compartir los resultados de una actividad exitosa con sus alumnos para que estos la puedan replicar,

- Vincular de forma novedosa su enseñanza con problemas de la disciplina y/o con la futura profesión del estudiante y,

- Promover el cambio en la perspectiva de comprender los temas planteados.

La actividad menos observada con un 32\% de los casos tiene relación con la oferta a los alumnos de distintas alternativas para elaborar los trabajos o tareas. 
Tabla 4. Frecuencia de indicadores de creatividad en la Universidad 2.

\begin{tabular}{|l|c|c|c|c|c|c|c|}
\hline \multicolumn{2}{|c|}{ Indicadores de creatividad } \\
\hline & CR1 & CR2 & CR3 & CR4 & CR5 & CR6 & CR7 \\
\cline { 2 - 9 } & Porcentaje & Porcentaje & Porcentaje & Porcentaje & Porcentaje & Porcentaje & Porcentaje \\
\hline Se observa & 74.0 & 68.0 & 84.0 & 74.0 & 32.0 & 53.0 & 74.0 \\
\hline $\begin{array}{l}\text { No se } \\
\text { reconoce }\end{array}$ & 26.0 & 21.0 & 16.0 & 26.0 & 42.0 & 16.0 & 21.0 \\
\hline $\begin{array}{l}\text { No } \\
\text { corresponde }\end{array}$ & 0.0 & 11.0 & 0.0 & 0.0 & 26.0 & 31.0 & 5.0 \\
\hline Total & 100.0 & 100.0 & 100.0 & 100.0 & 100.0 & 100.0 & 100.0 \\
\hline
\end{tabular}

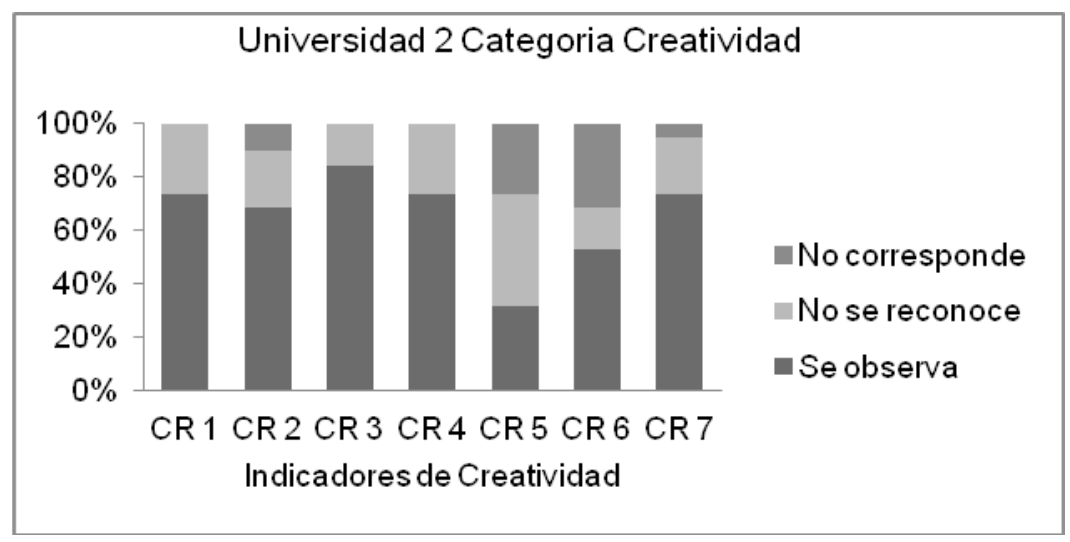

Fig. 2. Indicadores de creatividad y frecuencia observados en la acción docente.

\section{Universidad 3}

Para este grupo de docentes existen tres indicadores de creatividad vinculados con la transposición didáctica, los que se presentan con una mayor frecuencia en aula (ver tabla 5 y figura 3). El $81 \%$ de los docentes vincularon en forma novedosa su enseñanza con problemas de la disciplina ( $\mathrm{Cr} 7$ ), seguido de un 69\% que utiliza recursos estimuladores de la imaginación (Cr6) y que además promueven el cambio en la perspectiva de la comprensión de los temas planteados (Cr4). Con menor frecuencia el docente utiliza diversos medios para generar una clase original o creativa (Cr: 56\%). Asimismo, el ofrecer distintas formas de elaborar los trabajos se aprecia en una frecuencia menor (Cr5: 50\%). Finalmente la acción menos desarrollada fue compartir los resultados de una investigación o ponencia con sus alumnos (12\%). 
Tabla 5. Frecuencia de Indicadores de creatividad en la Universidad 3.

\begin{tabular}{|l|c|c|c|c|c|c|c|}
\hline \multicolumn{7}{|c|}{ Indicadores de creatividad } \\
\hline & CR1 & CR2 & CR3 & CR4 & CR5 & CR6 & CR7 \\
\cline { 2 - 8 } & Porcentaje & Porcentaje & Porcentaje & Porcentaje & Porcentaje & Porcentaje & Porcentaje \\
\hline Se observa & 12.0 & 56.0 & 25.0 & 69.0 & 50.0 & 69.0 & 81.0 \\
\hline $\begin{array}{l}\text { No se } \\
\text { reconoce }\end{array}$ & 69.0 & 38.0 & 69.0 & 31.0 & 19.0 & 25.0 & 19.0 \\
\hline $\begin{array}{l}\text { No } \\
\text { corresponde }\end{array}$ & 19.0 & 6.0 & 6.0 & 0.0 & 31.0 & 6.0 & 0.0 \\
\hline Total & 100.0 & 100.0 & 100.0 & 100.0 & 100.0 & 100.0 & 100.0 \\
\hline
\end{tabular}

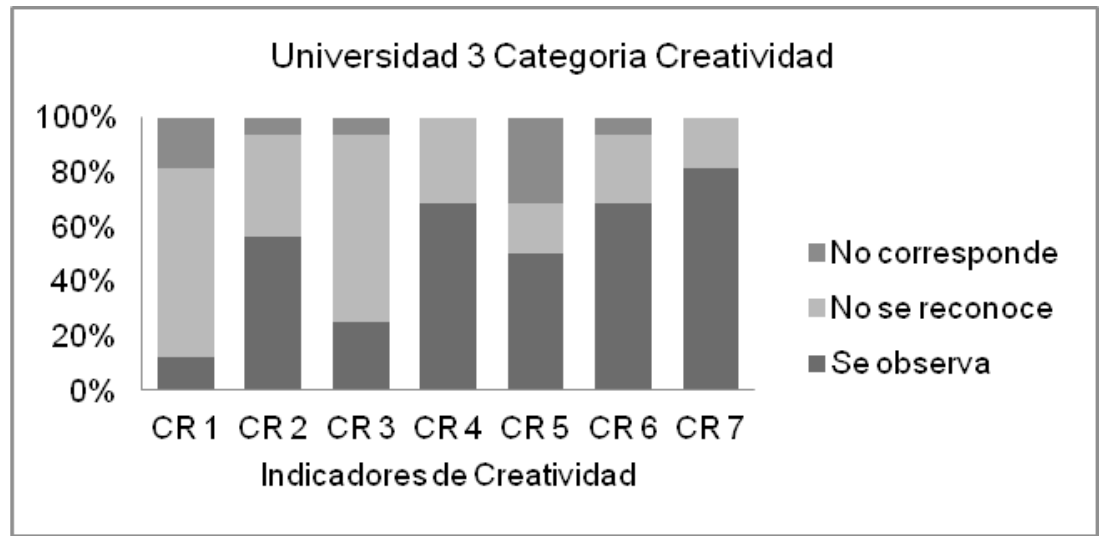

Fig. 3. Indicadores de creatividad y frecuencia observados en la acción docente.

Finalmente, para obtener una visión comparativa de la acción docente en las tres universidades de la muestra se consideró oportuno analizar la frecuencia de los indicadores de creatividad observados en las prácticas pedagógicas vinculada, dicha frecuencia, al enfoque de la transposición didáctica.

\section{Análisis global de los resultados}

Al igual que el análisis anterior, este estudio comparativo de naturaleza global se realizó con el fin de hallar tendencias en dicha muestra consultada pues se trata de encontrar una imagen global de la problemática en estudio.

\section{$C R$ 1: Compartir resultados de una actividad exitosa (ej. investigación, ponencia).}

Para este indicador de creatividad las frecuencias observadas fueron muy diferentes en las tres universidades. Tan solo el $12 \%$ de los docentes de la universidad 3 comparten los resultados de una actividad exitosa con sus alumnos siendo, asimismo, la frecuencia más baja observada en los docentes de la muestra. Lo anterior contrasta con lo que ocurre en la universidad 2, en la que el $74 \%$ de sus docentes evidencia realizar esta acción en sus prácticas de aula. En el caso la universidad 1 este valor es de un 42\% (véase figura 4). Elisondo (2015) indica que en las escuelas y universidades el cuerpo estudiantil puede vivenciar la pasión con que enseñan algunos docentes y esto parece ser un condicionante importante en la construcción de contextos creativos de enseñanza y aprendizaje. Esta pasión exhibida y transmitida invita en ocasiones a los estudiantes a adentrarse en ciertos campos de conocimiento y, los docentes, generalmente, suelen orientar en este camino. 
CR 2: El profesor utiliza diversos medios para generar una clase original e innovadora para sus alumnos.

Entre el 56\% y el 68\% de los docentes observados se preocupan por utilizar en sus aulas una variedad de recursos que les permiten generar una clase original para sus alumnos. En las tres universidades, por tanto, este indicador se observa con una frecuencia bastante similar (véase figura 4). El mismo permite reconocer la preocupación de los docentes por intentar motivar a los estudiantes mediante el uso de diferentes recursos de apoyo didáctico a fin de potenciar el desarrollo creativo de aquellos (De la Torre y Violant, 2006). Este hecho revela la utilización de mecanismos asociados a la transposición didáctica y a la búsqueda de una nueva organización conceptual del saber experto disciplinario en una nueva versión del saber didáctico que se ofrece en el aula (Chevallard, 1991).

\section{CR 3: El docente plantea preguntas divergentes o fecundas en sus resultados.}

Los sujetos de la universidad 3 en muy pocas ocasiones plantean preguntas divergentes (25\%). Esto contrasta con lo que ocurre en las universidades 1 y 2 donde el $84 \%$ de los docentes de ambas universidades plantean desafíos y preguntas divergentes a sus estudiantes, situación asociada a este indicador de creatividad observado (véase figura 4) donde es posible reconocer elementos asociados a una eventual transposición didáctica del docente universitario. Por cierto esta situación permite afirmar que, en algunas ocasiones, el sistema universitario y los aspectos externos pueden favorecer o dificultar la puesta en marcha de acciones educativas que fomenten y potencien la creatividad de su alumnado en la universidad.

Cabe señalar que en el ámbito de la educación superior deben crearse espacios de apertura a un pensamiento divergente que permita a los estudiantes y futuros profesionales enfrentar los problemas de una manera creativa.

\section{CR 4: El docente promueve el cambio en la perspectiva de comprender los temas planteados.}

Uno de los aspectos en los que existe una gran unanimidad es el hecho de que los docentes promueven el cambio en la perspectiva de comprender los temas planteados en clases. Por tanto, es posible observar esta acción entre el $69 \%$, el $74 \%$ y el $79 \%$ de los sujetos de la universidad 1, 2 y 3 respectivamente (véase figura 4). Detrás de dicha actitud docente es posible reconocer una mayor vigilancia epistemológica sobre el saber didáctico pues se reconoce una eventual obsolescencia de dicho conocimiento en la comprensión de ese saber experto que es transferido a la formación profesional (Chevallard, 1991).

Este cambio en la perspectiva de comprender los temas planteados es considerado un estímulo al desarrollo de la creatividad y puede suplir otras opciones más complejas de implementar (Alonso, 2001).

\section{CR 5: El profesor ofrece distintas formas o alternativas de elaborar los trabajos por hacer.}

Curiosamente, a diferencia del indicador anterior, la frecuencia observada desciende notoriamente en las universidades 1 y 2 (32\%) y tan solo se manifiesta en la mitad de los docentes de la universidad 3. Parece ser que se entregan alternativas para la comprensión de los temas tratados en clases pero no así muchas opciones o alternativas a la hora de elaborar trabajos o tareas en la asignatura dada (véase figura 4). Por cierto, hay una notoria diferencia entre elementos didácticos y evaluativos en la acción docente del profesor universitario que deben destacarse para un eventual y futuro perfeccionamiento docente. 
A pesar de que son muchas las variables que influyen en la potenciación de la creatividad puede reconocerse la importancia de aspectos organizativos tales como el número de alumnos y la infraestructura disponible en las universidades en tanto factores facilitadores o inhibidores de la conducta creativa. Es así cómo a mayor número de alumnos más complejo es ofrecer a los mismos una enseñanza basada en la innovación, la originalidad y la resolución de problemas. Esto se debe, posiblemente, a que al docente le supone una sobrecarga de trabajo dado que si tiene un gran número de alumnos o grupos de trabajo y quiere mantener un seguimiento de los avances y progresos de los mismos para optimizar sus resultados no puede hacerlo si no cuenta con apoyo institucional (Martínez y González, 2009).

Frente a lo anterior es posible que el docente evite el plantear distintas maneras para evaluar los trabajos debido a que los estudiantes no valoran ni aprovechan positivamente estos espacios de autonomía (Elisondo, 2015), y a que resulta escaso el uso hecho de los espacios de libertad y los intersticios que se suelen ofrecer en las clases, las tareas y las propuestas educativas.

Si bien existen evidencias de que la autonomía, la libre elección de temas y actividades así como las tareas complejas y flexibles promueven la creatividad, nuestras investigaciones y experiencias muestran que muchas veces estas particularidades de los contextos no son valoradas positivamente ni aprovechadas por los estudiantes para el desarrollo de pensamientos alternativos y de procesos creativos.

\section{CR 6: El profesor utiliza recursos estimuladores de la imaginación y la fantasía.}

La manifestación de este indicador no presenta grandes diferencias entre los docentes de las tres universidades regionales. El 63\% de los docentes de la universidad 1, la mitad de los docentes observados de la universidad 2 y el $69 \%$ de los docentes de la universidad 3 utilizan recursos estimuladores de la imaginación y la fantasía (véase figura 4).

En este aspecto el docente universitario se preocupa por utilizar procedimientos didácticos que potencien el uso de la imaginación para apoyar una mejor comprensión de sus materias de aprendizaje, en el entendido de que una mayor variedad de elementos de apoyo al aprendizaje estudiantil da oportunidad para acceder al saber experto que intenta desarrollarse mediante este saber didáctico inserto en dichas actividades pedagógicas.

$C R$ 7: El profesor vincula de forma novedosa su enseñanza con problemas de la disciplina y/o con la futura profesión del estudiante.

La frecuencia observada en las tres universidades es muy similar. En las aulas de las tres universidades se observa que el profesor vincula de forma novedosa su enseñanza con la futura profesión de sus estudiantes en un alto porcentaje: $74 \%$ en universidades 1 y 2 y $81 \%$ en universidad 3 (véase figura 4). Los docentes universitarios conectan frecuentemente la enseñanza con su futura experiencia profesional. De este modo, la apertura a la experiencia y los entornos abiertos promueven el desarrollo de procesos creativos (Elisondo, 2015).

Por cierto, en este aspecto observado en las aulas universitarias se destaca una preocupación mayor por ofrecer una mejor perspectiva sobre la naturaleza epistemológica del saber didáctico que se ha seleccionado para una formación profesional específica. De este modo se invita a transcender este conocimiento logrado en un ambiente específico por otro de mayor complejidad a fin de provocar un mayor interés por el origen 
epistemológico de tales saberes curriculares que caracterizan esta formación profesional, así como a insertarse en un nuevo ambiente disciplinario que puede ser de frontera en dicho ámbito profesional o científico.

Todo lo anterior permite reconocer que se pretende generar una nueva transposición epistemológica desde el saber didáctico a un saber experto; interesante cuestión gnoseológica para el desarrollo del proceso científico y creativo que se procura alcanzar en esta sociedad del conocimiento.

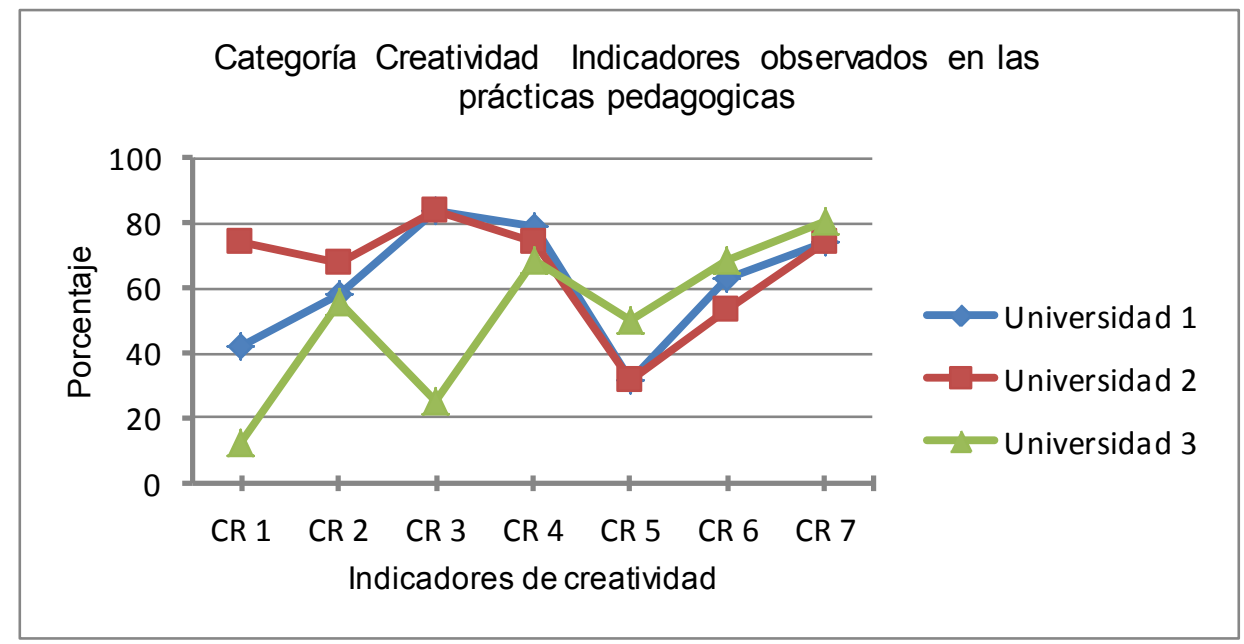

Fig. 4. Indicadores de creatividad observados en tres universidades regionales.

\section{A modo de conclusiones y desafíos futuros}

Al centralizar este estudio en la actuación docente y en los principales mecanismos didácticos utilizados por un profesor universitario se ha buscado caracterizar el proceso de transposición didáctica en función de algunos factores asociados al enfoque creativo de la educación. De este modo puede afirmarse que la transferencia didáctica es un vehículo promotor de la creatividad en la enseñanza superior, aun cuando no se tiene certeza de si existe como creencia, norma o patrón de la conducta pedagógica de un docente. Por cierto, esta problemática será materia de otro estudio.

Los docentes universitarios están conscientes de que sus alumnos una vez que egresen deberán resolver problemas y que su resolución implica recurrir a la creatividad. En concordancia con este planteamiento Duarte (1998) asegura que una de las primeras tareas a las que se enfrenta el egresado es solucionar problemas, problemas que distan de los enunciados teóricos de sus textos y que implican la utilización de algo más que su memoria y su comprensión. En esos momentos el nuevo profesional debe recurrir a su ingenio, originalidad, inventiva y flexibilidad, es decir, a su creatividad para solucionar un problema profesional.

Las diferencias que han podido detectarse responden a las realidades particulares de cada institución educativa. Es así como en relación a las estrategias y sus contextos De la Torre y Violant (2013) señalan que la realidad contextual es, sin duda, un elemento clave por cuanto sitúa la teoría y la acción en la realidad concreta donde confluyen decenas de variantes. La valoración del contexto, tal vez, sea el componente más sustantivo y esencial de la estrategia creativa frente a otros conceptos mediadores tales como método, procedimiento, técnica, entre otros, en los que predomina la secuencia encadenada. El 
contexto de la estrategia creativa es el referente de partida, del proceso y de llegada, pero que supone un buen manejo disciplinario del conocimiento necesario para su comprensión e intervención profesional en dicho ámbito.

En estos contextos universitarios debe considerarse el número de alumnos por curso, la infraestructura y el curriculum como factores clave que pueden potenciar o inhibir el desarrollo y la manifestación de la creatividad en los espacios educativos. La creatividad depende de interrelaciones entre sujetos y contextos, por lo tanto es importante advertir cómo se configuran los contextos con los que interactúan las personas. Además de los contextos en sí importa cómo estos son percibidos, interpretados y reconstruidos por los sujetos en un aula (Elisondo, 2015).

Se manifiesta la necesidad de construir entornos educativos creativos y los docentes se hacen eco de esta necesidad y de la responsabilidad que les compete en la generación de estos entornos. Sin embargo, también son conscientes de las limitaciones institucionales existentes para que la creatividad y sus manifestaciones perduren y se instauren en la universidad.

Esta labor no responde a eventos aislados y, tal como señala Elisondo (2015) la creatividad y las innovaciones no son eventos casuales ni fortuitos, son acciones sostenidas en el tiempo, sustentadas en decisiones y perspectivas nuevas acerca de los fenómenos. Es necesario invertir tiempo y esfuerzo en construir nuevos contextos educativos que den cabida a la creatividad, cuya naturaleza se encuentra asociada a una concepción revisada del saber disciplinario que le es propio y específico para esta formación profesional. Este desafío se vincula al enfoque de la transposición didáctica y sus mecanismos epistémicos (Villalobos Claveria y Melo Hermosilla, 2019).

Al formular estos desafíos se pretende responsabilizar a la institución educativa como el agente educativo ausente en la promoción de una cultura de la creatividad en la educación universitaria.

Por último, las particularidades del quehacer didáctico del profesor universitario, en tanto objeto de estudio e investigación, pueden ayudar a formalizar nuevos programas de apoyo a la formación docente que incluyan acompañamiento al profesor en sus innovaciones didácticas y desarrollo de una cultura de revisión permanente de su quehacer. Al mismo tiempo se abre un camino para fomentar la investigación en docencia universitaria bajo la mirada de nuevos paradigmas epistemológicos, metodológicos y didácticos en los que el enfoque de la transposición didáctica puede ser un factor promotor de dichos cambios. 


\section{Referencias bibliográficas}

Alonso, I. \& Arandia, M. (2014). Aprender creando: “Factoría Creativa" en las aulas universitarias. REDU. Revista de Docencia Universitaria, 12 (1), 443-468. https://polipapers. upv.es/index.php/REDU/article/view/6419/6483

Alonso Monreal, C. (2001): Qué es la creatividad. Madrid: Biblioteca Nueva, S.L.

Basto-Torrado, S. P. (2011). De las concepciones a las prácticas pedagógicas de un grupo de profesores universitarios. Magis. Revista Internacional de Investigación en Educación, 3(6) 393-412. https://doi.org/10.11144/Javeriana.m3-6.dcpp

Boden, M. (1994). La mente creativa: mitos y Mecanismos. Barcelona: Gedisa. http://www.scielo.org.co/scielo.php?script=sci_nlinks\&ref=000160\&pid=S0123$1294200800020001200006 \& \operatorname{lng}=\mathrm{es}$

Bolívar Botía, A. y Bolívar Ruano, M. R. (2012). La didáctica en el núcleo del mejoramiento de los aprendizajes. Entre la agenda clásica y actual de la Didáctica. Revista Perspectiva Educacional, 50(2), 3 - 26. http://www.perspectivaeducacional.cl/index.php/peducacional/ article/viewFile/38/18

Brousseau, G. (2011). La théorie des situations didactiques en mathématiques, Éducation et didactique [en línea], (5), 1. http://journals.openedition.org/educationdidactique/1005; DOI: https://doi.org/10.4000/educationdidactique.1005

Bunge, M. (1996). La ciencia. Su método y su filosofía. Editorial Sudamericana, Buenos Aires. https://users.dcc.uchile.cl/ cgutierr/cursos/INV/bunge_ciencia.pdf

Chevallard, Y. (1985). La transposition didactique - du savoir savant au savoir enseigné. $2^{\text {ème }}$ édition (1991). Un exemple de transposition didactique de Yves Chevallard et Marie-Alberte Johsua. Grenoble: La Pensée Sauvage. http://www.numdam.org/item/?id=PSMIR_1991 $\underline{\text { S6 } 39 \_0}$

Corbalán, J., Martínez, F. y Donolo, D. (2003). Manual CREA. Inteligencia creativa. Una medida cognitiva de la creatividad. Madrid: TEA. http://www.web.teaediciones.com/Ejemplos/ CREA-MANUAL-2015 extracto.pdf

Csikszentmihalyi, M. (1998). Creatividad. El fluir de la psicología del descubrimiento y la invención. Barcelona: Paidós Ibérica. https://campus.fundec.org.ar/admin/archivos/ Mihaly_2.pdf

De Camilloni, A., Cols, E., Basabe, L. y Feeney, S. (2007). El saber didáctico. Buenos Aires: Paidós.

De la Torre, S. \& Violant, V. (2013). Estrategias creativas en la enseñanza universitaria. Creatividad y sociedad. http://www.ub.edu/sentipensar/pdf/saturnino/estrategias creativas universitaria.pdf

De la Torre, S. y Violant, V. (2006), (coord. y dir.). Comprender y evaluar la creatividad, vol. 1. Málaga, España: Aljibe. 
Drucker, P. (1992). La sociedad poscapitalista. Editorial Sudamericana, Buenos Aires. http://transicionsocioeconomica.blogspot.com/2012/11/la-sociedad-poscapitalista-depeter.html

Duarte Briceno, Efrain. (1998). La creatividad como un valor dentro del proceso educativo. Psicologia Escolar e Educacional, 2(1), 43-51. https://doi.org/10.1590/S1413$\underline{85571998000100005}$

Elisondo, R. C. (2015). La creatividad como perspectiva educativa. Cinco ideas para pensar los contextos creativos de enseñanza y aprendizaje. Revista Actualidades Investigativas en Educación, 15(3), pp.1-23. DOI: http://dx.doi.org/10.15517/aie.v15i3.20904

Elmore, R.F. (2010). Mejorando la escuela desde la sala de clases. Santiago de Chile: Fundación Chile. Área de Educación. http://psicopedagogia.weebly.com/uploads/6/8/2/3/6823046/ libro_elmore.pdf

Finke, R.; Ward, T. \& Smith, S. (1996). Creative Cognition. Theory, Research and Applications. Cambridge: The MIT Press.

González, E. y Díaz, D. (2008). Desde el currículo hasta la didáctica o sobre la circulación de los saberes y sus controles en la universidad: un ejemplo en la enseñanza de la Medicina. latreia, 21(1), 83-93. http://www.scielo.org.co/pdf/iat/v21n1/v21n1a10.pdf

Hernández Sampieri, R.; Fernández Collado, C. \& Baptista Lucio, P. (2014). Metodología de la investigación. McGraw Hill, México. https://www.esup.edu.pe/descargas/ dep investigacion/Metodologia\%20de\%20la\%20investigaci\%C3\%B3n\%205ta\%20 Edici\%C3\%B3n.pdf

Jackson, P.W. (2009). La vida en las aulas. Morata, Madrid. http://www.terras.edu.ar/ biblioteca/6/PE_Jackson_Unidad_1.pdf

Jarauta Borrasca, B. y Medina Moya, J.L. (2012). Saberes docentes y enseñanza universitaria. En: Estudios sobre educación, (22), 179-198. http://diposit.ub.edu/dspace/ bitstream/2445/116036/1/609589.pdf

Lamo de Espinosa, E., González García, J.M. \& Torres Albero, C. (2007). La sociología del conocimiento y de la ciencia. Editorial Alianza, Madrid.

Litwin, E. (2010). El campo de la didáctica: la búsqueda de una nueva agenda. En A. Camilloni, M.C. Davini, G. Edelstein, E. Litwin, M. Souto y S. Barco, Corrientes didácticas contemporáneas (pp. 91-115). Buenos Aires, Argentina: Paidós.

Marina, J. (2011). Teoría de la inteligencia creadora. Barcelona: Anagrama. https://www. joseantoniomarina.net/libro/teoria-de-la-inteligencia-creadora/

Martínez, E. \& González, M. (2009). ¿La creatividad como competencia universitaria? La visión de los docentes. Revista de Formación e Innovación Educativa Universitaria 2(2), 101 114. http://refiedu.webs.uvigo.es/Refiedu/Vol2_2/REFIEDU_2_2_6.pdf 
Palomares, A. (2011). El modelo docente universitario y el uso de nuevas metodologías en la enseñanza, aprendizaje y evaluación. Revista de Educación, (355), 591-604. DOI 104438/1988-592X-RE-2011-355-038. http://www.revistaeducacion.educacion.es/re355/ re355 25.pdf

Pellón Arcaya, Mario, Mansilla Sepúlveda, Juan, \& San Martín Cantero, Daniel. (2009). Challenges to the Didactic Transposition and Knowledge of the Content in Teaching of Anatomy: Obstacles and Projections. International Journal of Morphology, 27(3), 743-750. https://dx.doi.org/10.4067/S0717-95022009000300018 https://scielo.conicyt.cl/scielo. php?script=sci_arttext\&pid=S0717-95022009000300018\&lng=en\&nrm=iso\&tlng=en

Pérez, L., Massón Cruz, R.M. y Torres Miranda, T. (2020). La formación profesional pedagógica del profesor universitario. Estudio comparado de experiencias universitarias. Revista Cubana de Educación Superior, 39 (1). Recuperado de: http://scielo.sld.cu/scielo. php?script=sci_arttext\&pid=S0257-43142020000100004\&lng=es\&tIng=es.

Perrenoud, P. (2010). Développer la pratique réflexive dans le métier d'étudiant. Professionnalisation et raison pédagogique. Paris: ESF éditeur (5. ${ }^{a}$ edición).

Sternberg, R., \& Lubart T. (1997). La creatividad en una cultura conformista. Un desafío a las masas. Madrid: Paidós.

Tardif, M. (2004). Los saberes del docente y su desarrollo profesional, Madrid: Narcea. http:// pdfhumanidades.com/sites/default/files/apuntes/TARDIF\%20-Los-Saberes-Docentes-ysu-desarrollo-profesional-pdf.pdf

Unesco, (2005). Hacia las sociedades del conocimiento: informe mundial de la UNESCO. Paris. http://www.lacult.unesco.org/docc/2005_hacia_las_soc_conocimiento.pdf

Villalobos Claveria, A. \& Melo Hermosilla, Y. (2019). Narrativas docentes como recurso para la comprensión de la transferencia didáctica del profesor universitario. Revista Formación Universitaria, vol. 12 (1), 121-132. https://dx.doi.org/10.4067/S0718-50062019000100121

\section{(cc) BY}

Este artículo está sujeto a una licencia internacional Creative Commons Attribution 4.0.

\section{${ }^{*} \mathrm{y}^{* *}$ Universidad de Concepción (Chile)}

${ }^{1}$ Doctor en Educación, Universidade Federal do Rio Grande do Sul (Brasil). Magister en Educación, mención Curriculum y Profesor de Filosofía, Universidad de Concepción (Chile). Profesor Asociado, departamento de Ciencias de la Educación, Facultad de Educación, Universidad de Concepción (Chile).

2 Doctora en Educación, Magister en Educación, mención Curriculum y Profesora de Francés, Universidad de Concepción (Chile). Profesora Asociada, departamento de Curriculum e Instrucción, Facultad de Educación. Universidad de Concepción (Chile). 


\section{Notas}

Aprobación final del artículo, editora responsable Mag. Verónica Zorrilla de San Martín

Contribución de autoría: Ambos autores han contribuido en partes iguales a la realización del citado artículo, tanto en la concepción del trabajo científico como en la recolección, interpretación y análisis de datos, la revisión y aprobación del manuscrito.

' Este artículo forma parte de los resultados del proyecto FONDECYT n 1130803: "El proceso de transferencia didáctica del docente universitario no pedagogo en la formación profesional superior. Un estudio de caso". 